Orthopäde $2021 \cdot 50: 3$

https://doi.org/10.1007/s00132-020-04030-3

(c) Springer Medizin Verlag GmbH, ein Teil von Springer Nature 2020

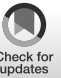

\title{
B. Greitemann
}

Klinik Münsterland, RehaKlinikum Bad Rothenfelde, DeutscheRentenversicherung Westfalen, Bad Rothenfelde, Deutschland

\section{Technische Orthopädie}

anzunähern. Auch hier sind Kombinationen mit osseointegrativen Verfahren derzeit eine höchst interessante Alternative.

Das Erkennen von Indikationen für notwendige Stumpfkorrekturoperationen ist eine wesentliche Voraussetzung, um Patienten zu helfen, die ansonsten nicht zufriedenstellend mit traditionellen Prothesenschäften versorgt werden können. Diese pure ärztliche Aufgabe beinhaltet ein ethisch sehr verantwortungsvolles Abwägen zwischen Operationsrisiko für die meist durchblutungsgestörten Patienten und funktionellem Zugewinn durch optimierte Stumpfbelastbarkeit. Diejenigen, die diesen Artikel intensiv lesen, werden hieraus zudem wichtige Informationen zur primären amputationschirurgischen Vorgehensweise entnehmen können.

Die beiden letzten Artikel der Serie beinhalten die prothetische Versorgung an der oberen und unteren Extremität. Michael Schäfer stellt die High-End-Versorgung bei armamputierten Patienten in Deutschland dar. Hier hat sich technisch eine Revolution entwickelt. Hände mit Einzelbewegungen von Fingern, Pattern Recognition, die es dem Anwender auch endlich ermöglicht, funktionell schnelle Greifbewegungen durchzuführen und die komplexe Bewegungsmuster unterstützen, sind heute in der Alltagsversorgung möglich. Der Artikel von Brückner und Gawron stellt hierzu als Pendant den Behandlungspfad der prothetischen Versorgung an der unteren Extremität auf hohem Niveau dar. Erstmals ist es hierbei gelungen, mit den beschriebenen Behandlungspfaden auch einen klaren Ablaufalgorithmus in der Verordnung nach funktionellen Kriterien zu erarbeiten. Diese Arbeit wurde im Wesentlichen unterstützt durch die Deutsche
Gesellschaft für interprofessionelle Hilfsmittelversorgung e. V. (DGIHV), einem Dachverband, bei dem sich Leistungsanbieter, Hilfsmittelhersteller und Fachverbände zu einer gemeinsamen Plattform zusammengeschlossen haben.

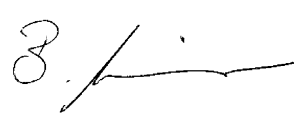

Prof. Dr. B. Greitemann

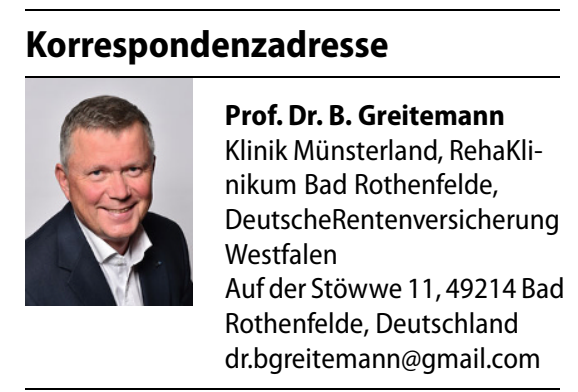

Interessenkonflikt. B. Greitemann gibt an, dass kein Interessenkonflikt besteht. 